\title{
De l'analyse des pratiques à la construction d'un modèle d'évolution des systèmes d'élevage : application à la région Nordeste du Brésil
}

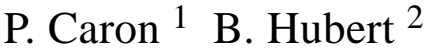

\begin{abstract}
Mots-clés
M éthode d'élevage - Modèle - Analyse de système - Conduite d'élevage Nordeste - Brésil.
\end{abstract}

\begin{abstract}
Résumé
Dans cet article est décrite une démarche méthodologique visant à comprendre l'évolution régionale de systèmes d'élevage à partir de l'analyse des pratiques des éleveurs. Après avoir présenté le cadre conceptuel dans la première partie, la méthodologie utilisée pour mettre en œuvre une telle démarche est décrite dans la seconde partie. Elle est illustrée, dans la troisième partie, par une étude de cas portant sur l'analyse des pratiques d'un éleveur de la région Nordeste du Brésil. Enfin, les auteurs ont développé leur démarche en proposant et discutant un modèle d'évolution des systèmes d'élevage dans cette région, construit à partir de l'analyse comparative des pratiques et des stratégies des éleveurs. L'identification et l'interprétation du changement ont requis I'analyse explicite de processus aussi bien locaux que globaux et l'intégration de différents pas de temps. Pour ce faire, une attention particulière a été accordée aux pratiques territoriales des systèmes d'élevage pour rendre compte de l'empreinte de l'espace et permettre l'intégration, dans I'analyse, d'échelles spatiales qui avaient un sens au regard du changement. A partir de l'analyse des pratiques, l'identification des modalités et des facteurs d'évolution des activités d'élevage a reposé sur un travail de modélisation dont les résultats sont discutés.
\end{abstract}

\section{INTRO DUCTION}

L'objectif de cette étude a été d'analyser les évolutions des activités d'élevage à l'échelle d'une région de vastes dimensions, qu'elle corresponde à une réalité administrative ou agro-écologique, en intégrant les dimensions techniques, économiques, sociales et spatiales qui interviennent dans les processus décisionnels. Pour identifier et interpréter le changement, les auteurs se sont intéressés à la manière dont les éleveurs géraient leur exploitation au sein d'une petite région et d'un environnement local à chaque fois particuliers. Ils ont cherché à rendre intelligibles les activités d'élevage et la façon dont elles étaient menées et se transformaient, du fait de l'adaptation par l'éleveur de ses projets aux évolutions de la situation dans laquelle il agissait.

1. Centre de coopération internationale en recherche agronomique pour le développement, département Territoires, environnement et acteurs (Cirad-tera), TA 60/15, 34398 Montpellier Cedex 5

2. Institut national de la recherche agronomique, département Systèmes agraires et développement (Inra/sad), 147, rue de l'Université, 75338 Paris Cedex 07
La recherche a été mise en œuvre dans la zone semi-aride de la région Nordeste du Brésil (figure 1). Les activités d'élevage y sont prédominantes, à l'exception de celles liées au développement local de l'irrigation. En considérant le troupeau comme le niveau pertinent de la gestion technique d'un éleveur, la recherche a été centrée sur les pratiques de constitution et de conduite des troupeaux.

Comme l'affirment Bonnal et coll. (1) dans la région centrale du Brésil, l'animal est essentiellement une fraction de patrimoine biologiquement reproductible au début de l'existence de l'exploitation, puis il devient un facteur de production de plus en plus spécialisé. Le troupeau joue en fait plusieurs fonctions simultanément. Il est épargne de précaution, essentielle en univers risqué et en contexte d'inflation et de refuge en économie de biens réels. C'est un capital productif et un produit tout à la fois. La structure du troupeau représente un indicateur de la nature et de l'importance accordée à chacune de ces fonctions. Dans cette étude, ce n'est pas seulement la pluralité des fonctions qui a compté, mais aussi la façon dont celles-ci sont combinées de manière spécifique à tout moment, lors d'un cycle de vie ou d'un exercice annuel, ainsi que la façon dont l'exploitant privilégie telle ou telle fonction selon les 


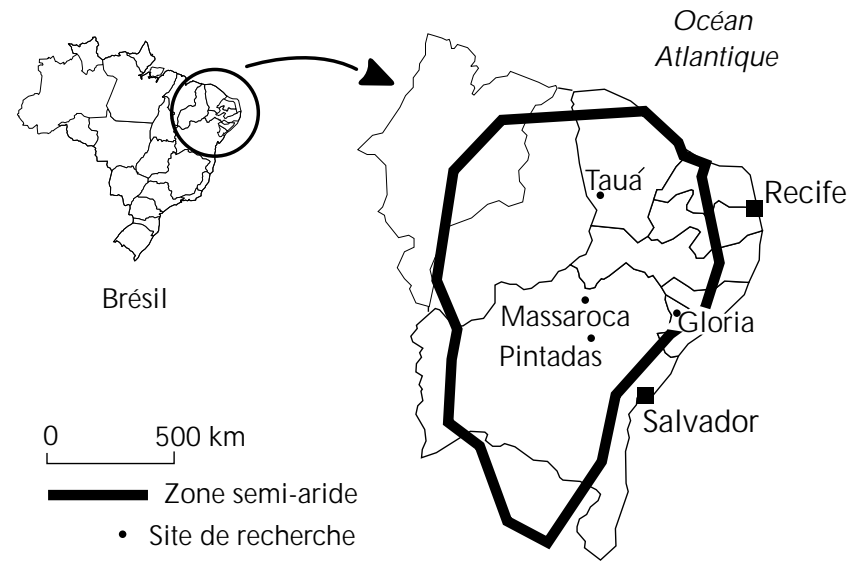

Figure 1 : les neuf Etats du Nordeste du Brésil et les sites de recherche.

conditions du moment. Les auteurs proposent de montrer en quoi cette plasticité est fondamentale, en procurant la flexibilité nécessaire à toute trajectoire sociale et en faisant de l'élevage le support conceptuel et matériel de stratégies évolutives complexes.

Le cadre de recherche conceptuel et méthodologique est d'abord présenté, illustré par une étude de cas portant sur l'analyse des pratiques d'un éleveur du Nordeste. Un modèle régional d'évolution des systèmes d'élevage, construit à partir de l'analyse comparative des pratiques et des stratégies des éleveurs pour la zone semi-aride du Nordeste du Brésil, est ensuite élaboré afin de relier ces évolutions individuelles à leur contexte collectif.

\section{- CADRE CONCEPTUEL} POUR L'ELABORATION D'UN MODELE D'EVO LUTION DES SYSTEMES D'ELEVAGE

Défini par des chercheurs zootechniciens, le concept de système d'élevage «s'inscrit dans un mouvement de pensée qui a touché de nombreuses disciplines techniques, et qui [...] commença à s'interroger dès les années soixante sur son efficacité pour le développement et sur la pertinence de ses propres pratiques de recherche... Les recherches sur les systèmes d'élevage [...] visent à définir un cadre conceptuel et méthodologique de portée générale, permettant à la fois : d'une part d'organiser l'ensemble des connaissances nécessaires pour analyser une situation d'élevage particulière et comparer entre elles des situations différentes ; d'autre part d'accéder aux représentations sur lesquelles s'appuient les divers acteurs qui ont à prendre des décisions en matière d'élevage » (15).

Pour cet auteur, un système d'élevage est « un ensemble d'éléments en interaction dynamique organisé par l'homme en vue de valoriser des ressources par l'intermédiaire d'animaux domestiques pour en obtenir des productions variées (lait, viande, cuirs et peaux, travail, fumure, etc.) ou pour répondre à d'autres objectifs ». Si les définitions des uns et des autres diffèrent, la plupart d'entre elles s'accordent, comme le propose Lhoste (20), pour reconnaître au système trois pôles : l'éleveur (ou, selon le niveau d'analyse retenu, le groupe social), le troupeau et le territoire pastoral. Elles s'accordent également pour admettre qu'il s'agit d'un système complexe piloté et finalisé. Pour relier pilotage et finalisation, les auteurs ont identifié ce qu'ils ont appelé des stratégies, lesquelles permettent à des observateurs externes de rendre intelligibles les projets des éleveurs à partir de l'étude de leurs pratiques.

\section{Modèle d'évolution des systèmes d'élevage par l'analyse comparative des stratégies d'éleveurs}

Les auteurs ont choisi de développer une démarche qui prenait en compte les projets des producteurs, sans pour autant ignorer que leurs objectifs étaient multiples, hiérarchisés et relatifs à des durées variables (5). Ces finalités n'étaient pas toujours explicites pour des tiers, en particulier pour des chercheurs. C'est pour cela que les auteurs proposent de rendre intelligibles ces objectifs, en analysant les stratégies que les producteurs ont élaborées pour les atteindre (29), c'est-à-dire, en procédant à une formalisation cohérente, pour les auteurs, des actions des producteurs (voir plus loin).

En effet, une stratégie n'est pas toujours formulée sous la forme d'un plan préconçu, conscient et finalisé. Mintzberg (21) distingue les stratégies prévues (intended strategy), qu'elles se concrétisent (l'auteur parle alors de stratégie délibérée - deliberate strategy) ou non, des stratégies émergentes (emergent strategy) lorsque des pratiques apparaissent sans préconception. Si les stratégies peuvent résulter d'intentions, elles peuvent aussi naître d'actions menées pas à pas qui, à leur tour, peuvent ultérieurement faire l'objet d'une stratégie délibérée. Ainsi, Chauveau (5) invite à la prudence et à ne pas « surinterpréter » de manière idéologique les capacités d'initiative et d'innovation.

Ce sont ces constructions stratégiques que les auteurs ont cherchées à formaliser. Une fois la diversité des stratégies des éleveurs mise en évidence, l'analyse comparative a été conduite selon deux axes :

- la comparaison des stratégies mises en œuvre par différents éleveurs dans une même petite région, afin d'apprécier l'influence du contexte local et des moyens de production disponibles, ainsi que la diversité des choix stratégiques ;

- la comparaison des stratégies mises en œuvre dans différentes petites régions, afin de préciser l'influence des contextes locaux, les similitudes et les spécificités engendrées par chacun d'entre eux.

Ces analyses comparatives ont permis d'élaborer une typologie des systèmes d'élevage et un modèle régional d'évolution des systèmes d'élevage et des exploitations agricoles. La modélisation ne constituait pas ici une démarche normative (18). Il s'agissait de la construction de modèles, compris comme des « représentations intelligibles artificielles, symboliques, des situations dans lesquelles nous intervenons [...] représentation artificielle que l'on construit dans sa tête » (19). Le modèle peut ensuite être « appliqué aux situations à étudier et permet d'en donner rapidement des représentations » (14). Le modèle proposé ici rend compte des modalités des trajectoires d'exploitation et de transition d'un type à l'autre, ainsi que des orientations stratégiques et des conditions qui président à ces évolutions.

\section{Pour caractériser les stratégies, l'observation des pratiques et l'élaboration de modèles de comportement pour l'action}

Ni le projet de l'éleveur, « ni ses objectifs, ni la manière dont il prend ses décisions, ne sont aisés à saisir pour un observateur extérieur » (17). L'action concrète de l'éleveur, acteur et décideur, se traduit par la mise en œuvre de pratiques. C'est alors l'interprétation d'observations des pratiques et d'entretiens sur leurs motivations qui permet de « comprendre comment les éleveurs prennent leurs décisions, à partir de quelles informations et par quelles actions, et pour viser quels objectifs » (9). 
Pour caractériser les stratégies, il a semblé intéressant, dans un premier temps, d'expliciter le cheminement décisionnel dont les pratiques découlaient (8). « Il ne s'agit pas de reconstruire un modèle de l'ensemble du système [...], mais plutôt de partir des heuristiques mises en œuvre pour caractériser les principales informations traitées, portant sur l'état du système, et qui sont à l'origine des prises de décision d'ordre technique, ainsi que de formaliser ces dernières d'une manière intelligible. C'est ce que nous appelons la construction d'un modèle de comportement pour l'action » (10). Cette construction vise, pour le technicien ou le chercheur, à élaborer une représentation de la réalité qui rend compte de ce qui apparaît comme important pour l'éleveur dans la conduite du système. On cherche à rendre intelligible les décisions du producteur par la mise en évidence de cohérences révélées par son comportement. Cette formalisation permet de comparer les stratégies et les pratiques des producteurs et d'identifier des voies pour accompagner et favoriser leurs transformations.

Après avoir fait l'objet d'une première définition sous l'expression de « modèle général » (6), le concept de modèle d'action a été proposé par Sebillote et Soler (25) pour représenter l'organisation des prises de décisions des agriculteurs dans le cadre d'un processus récursif et adaptatif permanent : « l'acteur réajuste ainsi de manière conjointe ses finalités et son action sur la réalité. Il se constitue, pour ce faire, un véritable guide pour l'action, dont la représentation [par le chercheur] peut s'organiser autour d'un ou plusieurs objectifs généraux [...], d'un programme prévisionnel et des états-objectifs intermédiaires [...] et d'un corps de règles [...]. »

$\mathrm{Au}$ sein du corps de règles, Hubert et coll. (12) distinguent des règles générales et des règles circonstancielles : " les premières s'appliquent inéluctablement pour la satisfaction du projet ; elles traduisent la construction du système en opérations techniques concrètes, quels que soient les événements du moment. Les secondes, à l'inverse, sont activées par une information relative à l'état de certains des éléments du système : elles déclenchent conditionnellement des actions, qui connaissent différentes modalités ou dont la mise en œuvre peut être avancée ou retardée selon les conditions du moment. La formalisation de ces règles éclaire sur le système d'information utilisé par l'éleveur dans le processus d'auto-diagnostic qu'il met en œuvre pour réaliser ses différentes opérations techniques ». Il devient alors possible de repérer « des décisions remarquables qui scandent la conduite de la campagne annuelle » et d'identifier « des phases finalisées à partir du découpage issu des décisions remarquables ».

La mise en évidence de corps de règles définis à partir de l'observation des pratiques et la formulation de « modèles de comportement pour l'action » permettent de caractériser les stratégies des producteurs (12).

\section{MATERIEL ET METHODES}

\section{Des pratiques portant sur le troupeau, mais aussi sur le territoire}

Landais et coll. (16) proposent de classer les pratiques d'élevage en distinguant : (i) les pratiques d'agrégation, opérations de constitution des troupeaux et des lots ; (ii) les pratiques de conduite, « opérations effectuées par l'homme sur les animaux en vue d'assurer leur entretien et les mettre en condition de réaliser les performances de croissance, de reproduction, de production »; (iii) les pratiques d'exploitation «par lesquelles l'homme exerce un prélèvement »; (iv) les pratiques de valorisation des productions animales.
Ces pratiques concernent des opérations réalisées sur les animaux ou leurs produits. Or, les caractéristiques et les enjeux territoriaux, en particulier les dynamiques d'appropriation et de mise en valeur des ressources foncières, jouent souvent un rôle important dans le fonctionnement technique des systèmes de production. L'espace devient territoire grâce à l'action organisatrice de l'éleveur et les différentes portions de ce territoire, qu'elles soient en propriété individuelle ou collective, sont amenées à remplir des fonctions spécifiques dans le processus de production. «Une fonction est caractérisée par une affectation sur le territoire (une ou plusieurs parcelles peuvent y contribuer), une période dans l'élaboration de la production des animaux (la croissance, la lactation, la remise en état, etc.) et une phase dans le programme de complémentation du troupeau (la ration étant, exclusivement ou non, prélevée par les animaux). Trois grands groupes de fonctions d'une ressource fourragère peuvent ainsi être repérés : les fonctions directement liées à l'alimentation du troupeau (constitution de stocks et pâturage), celles liées aux conditions d'utilisation de certaines surfaces et aux fluctuations du climat (fonctions de régulation et de soudure) et des fonctions annexes (liées à la contention du troupeau, au travail de l'éleveur, etc.) » (9). La partie de territoire en question ne possède pas de fonction. Elle contribue au fonctionnement du système en assumant une ou plusieurs fonctions spécifiques à une date et pour une durée précises.

Le territoire pastoral de l'éleveur ne se réduit pas à un espace soumis aux pratiques d'élevage. Il est modelé, transformé, produit pour être amené à assurer de nouvelles fonctions. De façon à prendre en compte les dimensions spatiales de ces activités, des « pratiques territoriales » des systèmes d'élevage ont été également identifiées selon quatre catégories :

- l'agrégation territoriale qui consiste à intégrer de nouveaux espaces à l'exploitation, par l'appropriation de ressources collectives (usage pastoral, pose de clôtures) ou l'achat de terres, ou à abandonner des espaces (diminution de la main d'œuvre, distance au siège de l'exploitation, problèmes de fertilité du sol, vente) ;

- la transformation des couverts végétaux (défriche, installation de cultures, fourragères ou non, etc.) ;

- l'équipement du territoire de l'exploitation par la mise en place d'infrastructures (point d'eau, division d'une parcelle, parc de contention, types de clôtures, etc.) ;

- l'affectation d'une parcelle ou d'un parcours à un usage particulier à un moment donné et pour une période définie (production agricole, pâturage, infirmerie, mise en défens, cueillette de fruits, coupe de bois, etc.).

\section{Application à la région Nordeste du Brésil}

Le Nordeste constitue une des cinq régions administratives du Brésil ; il est composé de neuf états. La population de la zone semiaride $\left(937000 \mathrm{~km}^{2}\right)$, le Sertão, représente 38 p. 100 des 29 millions de nordestins (13).

Le Nordeste a mauvaise réputation : «Région problème [...] la plus pauvre du pays, la plus défavorisée » (27). Pour expliquer la situation, on invoque souvent la sécheresse. Mais la diversité physique invalide cet argument. Au-delà des conditions climatiques difficiles, la pauvreté du Nordeste est en fait liée au système latifundiaire d'origine. L'accès à la terre a été refusé à une grande partie de la population. La concentration des richesses aux mains d'une élite et le caractère excentré de l'économie ont rendu l'investissement rare (28). 
Les travaux de recherche ont été conduits de 1987 à 1995 dans quatre petites régions du Nordeste semi-aride (figure 1), afin d'analyser les techniques mises en œuvre par les éleveurs à la lumière et en tenant compte des spécificités locales : Massaroca et Pintadas (Etat de Bahia), Nossa Senhora da Gloria (Etat du Sergipe) et Tauá (Etat du Ceará). Les conditions y sont à chaque fois particulières. La localisation, les caractéristiques des ressources naturelles, l'histoire et les formes d'organisation sociale et politique sont contrastées. Les quatre études de cas présentent des situations qui recouvrent la diversité des formes d'élevage dans le Nordeste (7). L'élevage ultra extensif repose sur la valorisation de parcours ouverts à Massaroca, où l'aridité est plus marquée et les petits ruminants dominent. A Pintadas, l'élevage, essentiellement bovin, est extensif en surfaces clôturées, alors que l'activité agricole est très présente. L'élevage semi-intensif avec production fourragère irriguée dans les bas-fonds se développe à Tauá, mais co-existe avec les formes précédentes. A Nossa Senhora da Gloria, en zone de transition climatique et à proximité de grands centres urbains, la production de lait connaît un essor considérable.

Les études de cas n'ont aucune valeur de représentativité statistique, mais elles sont pertinentes vis-à-vis des questions soumises à la recherche. Ces petites régions ont été choisies pour un ensemble de raisons qui tiennent autant à la diversité des situations qui les caractérisent qu'à l'histoire du projet de recherche et à la contingence des travaux entrepris dans ce cadre. Elles font toutes partie du réseau de projets de développement local dans lequel s'est investi le projet d'Appui au développement de l'agriculture familiale du Nordeste. A ce titre, elles sont le lieu de dynamiques sociales ou institutionnelles fortes qui ont conduit les acteurs locaux (certains tout du moins) à solliciter un appui externe (3).

\section{Suivi d'une trentaine d'éleveurs}

Dans chaque localité, un éleveur a été sélectionné pour chacun des sous-types de la typologie structurelle d'exploitations préalablement élaborée, soit une trentaine au total (3). Chacun d'entre eux a fait l'objet d'un suivi d'une durée minimale d'un an.

Une première enquête approfondie réalisée à l'aide d'une grille ouverte, éventuellement en deux passages, a permis de caractériser la structure foncière de l'exploitation, la nature des activités agricoles et autres, les moyens de production mobilisables et la structure du troupeau. L'enquête a également porté sur les évolutions intervenues depuis l'installation de l'exploitant, en identifiant les changements majeurs qui ont marqué la trajectoire d'exploitation, qu'ils concernent le parcellaire et l'aménagement foncier, la structure de la famille, la migration, la reconversion d'une espèce à l'autre, etc.

Sur la base de ces informations, il a alors été demandé à l'éleveur de caractériser la chaîne de pâturage des différents lots de son troupeau au cours de l'année précédente et de justifier les choix opérés. Enfin, une prévision concernant la constitution des lots et la chaîne de pâturage pour l'année à venir était formulée.

Par la suite, un suivi mensuel a été mis en place. Chaque passage visait à recueillir les données relatives à l'évolution démographique du troupeau (naissances, décès, achats, ventes), à la constitution et à la conduite alimentaire (parcelles attribuées, supplémentation, etc.) des différents lots et à la nature et au montant des revenus et des dépenses. Chaque pratique a d'abord été caractérisée, puis validée avec l'éleveur qui était également interrogé sur les raisons d'agir ainsi ou de réfuter une alternative qui s'offrait à lui. Le tableau I illustre cette démarche par le cas d'un éleveur : quelles étaient les entités sur lesquelles il a agi, pourquoi et en fonction de quels critères ? C'est à partir de ces échanges que les auteurs ont rendu intelligibles pour eux-mêmes les règles de décision de l'éleveur. Le tableau II présente ainsi, pour le même éleveur, le contenu et les critères de décision pour chacune des huit classes de pratiques des systèmes d'élevage (de gestion du troupeau et de gestion de l'espace). C'est à partir de cet ensemble de données qu'il a été possible de décrire la cohérence fonctionnelle du système, en particulier celle attribuée aux différentes parties du territoire pastoral. L'éleveur était enfin invité à expliquer et à justifier tout changement, par rapport aux données recueillies le mois précédent, et tout écart avec la chaîne de pâturage annuelle imaginée en début de suivi.

En cas de suivi pluriannuel (Massaroca et Nossa Senhora da Gloria), les modifications apportées à l'espace pastoral étaient analysées et une nouvelle prévision concernant la chaîne de pâturage était formulée au début de chaque cycle annuel.

\section{- ETUDE DE CAS : VALDEMAR, ELEVEU R DANS LA ZONE SEM I-ARIDE DU NORDESTE DU BRESIL, A MASSAROCA}

Afin d'illustrer cette démarche, le cas d'un éleveur, Valdemar à Massaroca, est présenté. Il a été choisi parce qu'il illustrait une situation très évolutive, caractéristique des trajectoires de jeunes exploitants en dynamique d'installation dans le Nordeste. Il ne représentait pas les types d'exploitations plus anciennes et relativement établies.

\section{Histoire de Valdemar et de son exploitation}

Valdemar est né en 1954. Il est descendant du fondateur de la communauté de Lagoinha où il résidait, à $60 \mathrm{~km}$ au sud de Juazeiro dans l'Etat de Bahia (figure 1). Marié depuis 1981, il avait trois enfants en bas âge au moment de l'étude. A l'âge de 20 ans, il est parti travailler à São Paulo, dans le sud du pays, comme maçon, ainsi que l'avaient fait ses frères aînés. A l'inverse de ces derniers, l'accumulation qu'il a pu réaliser pendant la migration a été limitée. Le contexte n'était déjà plus aussi favorable.

Il possédait quand même un troupeau d'une quarantaine de caprins à son retour à Lagoinha, trois ans plus tard. Il a exploité une parcelle prêtée par sa mère et a travaillé dans la mine de cuivre de Caraiba Metais à une trentaine de kilomètres et comme maçon localement. En 1985, il a clôturé une première parcelle de 1,6 ha sur une terre de plusieurs dizaines d'hectares reçue de ses parents à proximité de sa maison, grâce au financement d'un projet public de développement. Les sols bruns non calciques pierreux et peu profonds du carrasco ne se prêtaient pas aux cultures. Il a mis en place une prairie de capim buffel (Cenchrus ciliaris) qui a fourni un stock fourrager de soudure pour son troupeau d'environ 25 caprins.

Il a continué de cultiver la parcelle de sa mère jusqu'en 1988, date à laquelle celle-ci l'a récupérée en échange d'une centaine d'hectares non clôturés de caatinga (végétation arborescente xérique et épineuse caractéristique du Nordeste). Cette surface se trouvait sur les régosols à « vocation agricole » (areias), en vis-à-vis du village, de l'autre côté de la rivière temporaire Barrinha. C'est là qu'il a décidé de mettre en valeur la terre, plutôt que sur les sols pauvres du carrasco. Grâce à un crédit du Comité des associations agropastorales de Massaroca (Caam), dont il a été l'un des premiers bénéficiaires, il a clôturé quatre hectares dont trois ont été défrichés et plantés. Il a privilégié les produits agricoles, ne disposant pas d'autres terres sur lesquelles il aurait pu planter du maïs, des haricots, du manioc et des pastèques. 
Tableau I

Critères de prise de décision de Valdemar relatifs à la chaîne de pâturage

Pratique

observée
Enquête sur les motivations

\section{Critères de prise} de décision
12 femelles allaitantes

(dont 8 primipares) dansla parcelle A (avril)

Femelles allaitantes et gravides dans la parcelle B (septembre)

Cabris dans la parcelle $A$ (septembre)

Chèvres dans la parcelle C (octobre)
$V$ : «Les chèvres ont utilisé le capim pendant une semaine. Après, les chèvres ont commencé à avorter, j'ai eu peur. J'ai commencé à distribuer le foin de pois d'Angole que je n'avais pas prévu d'utiliser avant septembre-octobre. Les huit chevrettes et une chèvre ont avorté. »

Q : «Après les avortements, pourquoi ne pas relâcher les femelles ayant avorté dans la caatinga ? Vous auriez économisé vos ressources fourragères ».

$\mathrm{V}$ : «Ce n'était pas possible. Elles étaient vraiment trop faibles. Après, je les ai même passées dans les restes de cultures (areias) pour les retaper ».

V : « J'ai rassemblé les femelles de la première et de la deuxième mise bas avec les femelles en fin de gestation, dans la parcelle areias pour utiliser les restes de cultures. Les récoltes étaient terminées depuis trois semaines et j'ai laissé un peu de manioc sur pied pour les animaux (les animaux déterrent eux-mêmes les racines). La parcelle de la Rodagem (A) était rasée, les animaux maigrissaient depuis 15 jours ».

Dans les areias, les femelles ont aussi utilisé la caatinga mise en défens. Six femelles ont mis bas dans cette parcelle.

Q : « Mais pourquoi avez-vous laissé les cabris dans la parcelle de la Rodagem où il n'y avait plus rien à manger? »

$V$ : « Ces cabris étaient sevrés, séparés de la mère qui les avait abandonnés ou qui était morte ; c'était des enjeitadas. Dans la parcelle des areias, il n'y avait que des restes de culture hauts (ricin, manioc, pois d'Angole). Les cabris tétant ont la possibilité d'utiliser quelques feuilles tombées sur le sol, le lait complète. Les cabris sevrés n'auraient profité que des débris sur le sol. J'ai préféré les laisser sur place et leur distribuer des graines de coton et du manioc en morceaux. J'ai également mis le cheval du voisin pour nettoyer le capim, c'est-à-dire pâturer les tiges dures laissées par les chèvres et favoriser une bonne repousse au moment des pluies ».

V : «Les restes de culture s'appauvrissaient. Un jour ou l'autre j'aurais été obligé d'acheter du concentré pour maintenir la lactation, mais aussi de faire un nouveau chargement d'eau. J'ai préféré passer rapidement les chèvres dans la parcelle de la maison où la mare communautaire est proche. Avec l'argent de l'eau économisé, j'ai pu acheter plus de concentré (graines de coton). J'ai rassemblé tous les animaux dans cette parcelle, la caatinga était trop faible. Je complémentais également avec du mandacaru* que j'allais cueillir tous les trois jours. C'était beaucoup de travail, j'avais aussi une portion de caatinga de la parcelle des areias à déboiser pour la prochaine mise en culture ».
Utilisation d'une parcelle proche de la maison pour une meilleure surveillance des mises bas et des jeunes.

État physiologique des femelles et état des ressources de la parcelle de la Rodagem (A).

Taille des animaux et état des ressources dans la parcelle des areias (B).

\section{État des} ressources. Coût de l'abreuvement.

D'après Paris, 1992, Mémoire de fin d'études, Eitarc/Cnearc, Montpellier, France

$\mathrm{V}=$ Valdemar $; \mathrm{Q}=$ question des auteurs

* Cactus de la caatinga : Cereus jamacaru

\section{Conduite du système de production}

\section{Pratiques d'organisation du territoire de l'exploitation}

A partir de ce moment-là, il a mobilisé tous les fonds disponibles pour accroître chaque année un peu plus la surface clôturée sur les areias (figure 2). Vente d'animaux, revenus de maçonnerie en saison sèche, un deuxième crédit du Caam en 1993, tout a été investi. En 1994, la surface clôturée sur les areias était de 14 ha, dont la moitié était défrichée et cultivée : maïs, haricots et manioc pour la consommation familiale et la vente éventuelle d'excédents, pastèques pour la vente. Valdemar a associé du capim buffel aux cul- tures. Cela lui a permis d'accroître le stock fourrager pour l'alimentation en saison sèche des animaux qui pâturaient alors les restes de culture et les graminées. Le manioc, bi-annuel, était protégé par une clôture. Après deux ou trois années de culture, le temps qu'il a fallu pour défricher une autre parcelle et délocaliser les cultures vivrières et de rente, la prairie était installée. Le Cenchrus ciliaris est apprécié des éleveurs en raison de sa résistance à la sécheresse et de la facilité d'installation des prairies. Les variétés utilisées possédaient une production importante de semences (environ $50 \mathrm{~kg} / \mathrm{ha}$ ) au fort pouvoir de germination. Les semences, très lourdes, restaient au pied des plants et germaient dès l'arrivée 


\section{Tableau II}

Pratiques du système d'élevage de Valdemar et critères de prise de décision

\begin{tabular}{|c|c|c|c|}
\hline \multicolumn{2}{|c|}{ Pratiques } & Caractéristiques & Critères de prise de décision \\
\hline \multirow{5}{*}{$\begin{array}{l}\text { Gestion } \\
\text { du } \\
\text { troupeau }\end{array}$} & Agrégation & $\begin{array}{l}\text { Constitution de lots, caractérisée par: } \\
\text { - complexité } \\
\text { - nombreux ajustements tactiques } \\
\text { - tri individuel des animaux }\end{array}$ & $\begin{array}{l}\text { - l'animal (espèce, sexe, âge ; état physique, physiologique } \\
\text { ou pathologique; niveau de production ; conduite } \\
\text { antérieure - presa, enjeitada, mateira) } \\
\text { - le troupeau, pour achat éventuel (taille du troupeau ; } \\
\text { liquidités monétaires ; objectifs d'amélioration génétique) } \\
\text { - la ressource végétale et la structuration de l'espace } \\
\text { (hauteur des strates fourragères; disponible fourrager } \\
\text { compte tenu des besoins en saison sèche ; type de clôture ; } \\
\text { présence d'un point d'eau) } \\
\text { - la supplémentation éventuelle (disponible fourrager ; } \\
\text { abreuvement hydrique ; disponible monétaire ; pari sur } \\
\text { la date d'arrivée des pluies) }\end{array}$ \\
\hline & Conduite & $\begin{array}{l}\text { Succession de phases de conduite alimentaire } \\
\text { marquées par les saisons: } \\
\text { - mise à l'herbe sur parcours non clôturé de } \\
\text { caatinga à l'arrivée des pluies (enjeitada et } \\
\text { mateira) } \\
\text { - surveillance des mises bas en enclos (presa) } \\
\text { - entretien sur surfaces clôturées à partir } \\
\text { du début de la saison sèche } \\
\text { - supplémentation éventuelle à l'aide } \\
\text { d'aliments industriels en fin de saison sèche }\end{array}$ & $\begin{array}{l}\text { - l'animal (comme précédemment) } \\
\text { - la ressource végétale et la structuration de l'espace } \\
\text { (comme précédemment) } \\
\text { - la supplémentation éventuelle (comme précédemment) }\end{array}$ \\
\hline & Exploitation & $\begin{array}{l}\text { Trois types de vente: } \\
\text { - groupée en début de saison sèche dans } \\
\text { une logique de rente } \\
\text { - régulière au cours de l'année pour } \\
\text { les besoins de trésorerie } \\
\text { - groupée en fin de saison sèche pour } \\
\text { les problèmes de trésorerie }\end{array}$ & $\begin{array}{l}\text { - prix de vente } \\
\text { - structure du troupeau } \\
\text { - état des animaux (espèce, âge, sexe, état d'engraissement) } \\
\text { - besoins monétaires }\end{array}$ \\
\hline & Valorisation & $\begin{array}{l}\text { Transformation pour l'autoconsommation } \\
\text { (salage et séchage) et vente sur pied }\end{array}$ & besoins de consommation familiale \\
\hline & Affectation & $\begin{array}{l}\text { A l'interface de la gestion du troupeau } \\
\text { et de la gestion de l'espace }\end{array}$ & $\begin{array}{l}\text { - état de la ressource végétale (offre fourragère et entretien } \\
\text { de la ressource) } \\
\text { - mise en défens avant les récoltes ou permanente (palme, } \\
\text { manioc...) } \\
\text { - l'animal (voir gestion du troupeau) } \\
\text { - la supplémentation (voir gestion du troupeau) } \\
\text { - localisation par rapport aux parcours et au siège } \\
\text { d'exploitation }\end{array}$ \\
\hline \multirow{3}{*}{$\begin{array}{l}\text { Gestion } \\
\text { de } \\
\text { l'espace }\end{array}$} & Agrégation & Clôture de parcours de caatinga & $\begin{array}{l}\text { - statut et héritage fonciers } \\
\text { - autres parcelles clôturées et choix de production } \\
\text { - disponibilité financière (fil de fer barbelé) } \\
\text { - type de sol } \\
\text { - possibilité de mise en place d'un point d'eau } \\
\text { - localisation et distance du siège d'exploitation }\end{array}$ \\
\hline & $\begin{array}{l}\text { Transformation } \\
\text { des couverts } \\
\text { végétaux }\end{array}$ & Défriche et mise en place de cultures & $\begin{array}{l}\text { - disponibilité en main d'œuvre familiale ou salariée } \\
\text { - type de sol } \\
\text { - choix de production }\end{array}$ \\
\hline & Equipement & $\begin{array}{l}\text { Principalement mise en place de points } \\
\text { d'eau pour l'abreuvement des animaux en } \\
\text { saison sèche }\end{array}$ & $\begin{array}{l}\text { - ressources naturelles } \\
\text { - capacité d'investissement ou opportunité d'aides publiques } \\
\text { - fonctions auxquelles contribue la parcelle dans le } \\
\text { calendrier fourrager (s'applique aussi aux parcs de } \\
\text { contention) }\end{array}$ \\
\hline
\end{tabular}


1976-1980 : migration à São Paulo

1981

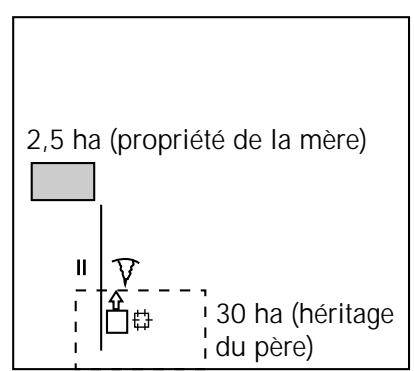

1985

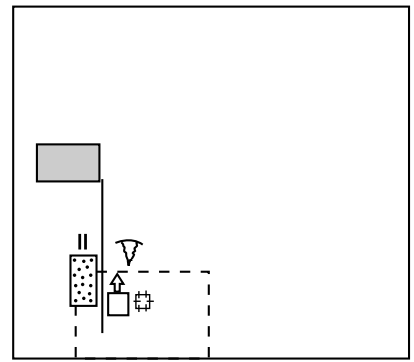

1989

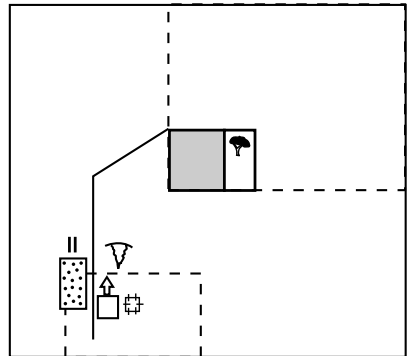

1994

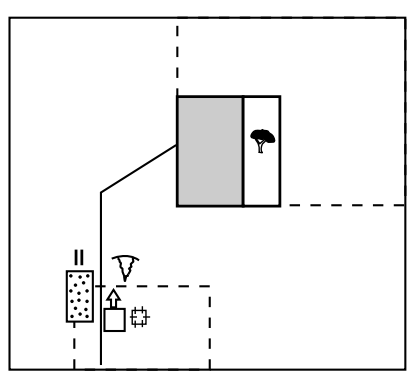

La mise en place de

L'installation avec un troupeau de 40 caprins

(27 ans)

cultures fourragères

(1,6 ha) sur des terres

ne permettant pas

l'agriculture (financée

par projet São Vincente)

25 caprins
II Mare

i Habitation

$\rightarrow$ Extension

$\backslash$ Piste

Parc de nuit

Surface clôturée

Parcours clôturé

Surface fourragère clôturée

Surface agricole clôturée

Terres en propriété

L'héritage de terres de

sa mère sur les régosols

(100 ha)

Clôture (4 ha) et mise en

culture (3 ha) grâce à

un crédit du Caam

50 caprins

Le projet

Augmentation des surfaces cultivées

et clôturées

Achat d'ovins

\section{Augmentation}

progressive de la

surface clôturée sur

régosols $(15,5$ ha,

dont 8,5 cultivés

et 7 non défrichés;

6,5 ha clôturés en 1992)

50 caprins (40 en 1992) des pluies. Compte tenu du caractère invasif de la graminée, la prairie est installée en quelques années avec un travail minime, hormis lors de la première année. Durant la phase d'invasion, l'association de cultures de rente et vivrières ont permis de rentabiliser l'investissement.

\section{Pratiques de gestion du troupeau}

La représentation spatiale des ressources fourragères a permis de caractériser les pratiques de gestion du troupeau sous la forme d'une chaîne de pâturage (figures 3 et 4 et tableau III). Sur celle-ci sont figurées les pratiques d'allotement, d'affectation des ressources végétales aux lots d'animaux, de conduite de la reproduction et les fonctions auxquelles chaque portion de territoire a contribué durant une période précise de l'année. Dans le cas de Valdemar, le territoire pastoral comprenait quatre unités - conséquence des priorités de l'éleveur en termes d'équipement - dont la fréquence d'utilisation a varié au cours de l'année, en fonction des saisons ainsi que des allotements. Ces derniers étaient liés à la reproduction du troupeau.

La structure d'ensemble était rudimentaire. Elle exprimait la faible marge de manœuvre dont Valdemar disposait pour s'adapter aux événements, que ceux-ci aient été prévisibles ou non. Une plus grande diversité de parcelles (taille, faciès de végétation, point d'abreuvement, distances, etc.) lui aurait offert une plus grande souplesse d'ajustement et lui aurait permis de répartir son troupeau en différents lots selon leur sensibilité, ainsi que le faisaient 


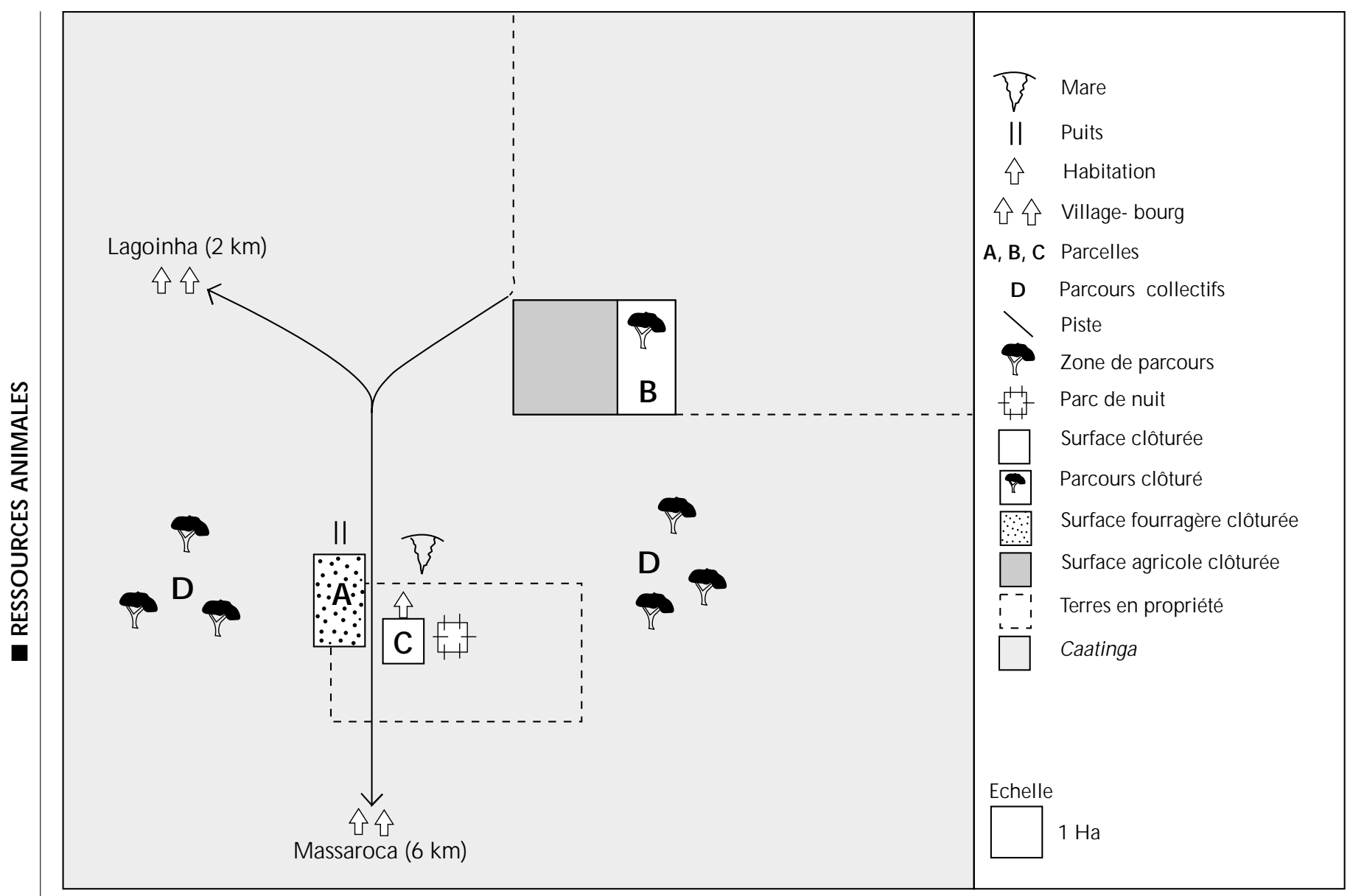

Figure 3 : territoire de l'exploitation de Valdemar en 1991.

d'autres éleveurs de Massaroca. Pour valoriser au mieux les ressources fourragères limitées, le nombre d'allotements était important. Seuls les principaux sont figurés sur la chaîne de pâturage.

Chaque parcelle assurait des fonctions différenciées au cours de l'année. La parcelle A contribuait successivement à des finalités différentes : infirmerie, maternité, production, soudure et flushing. Les fonctions auxquelles contribuait la parcelle B concernaient

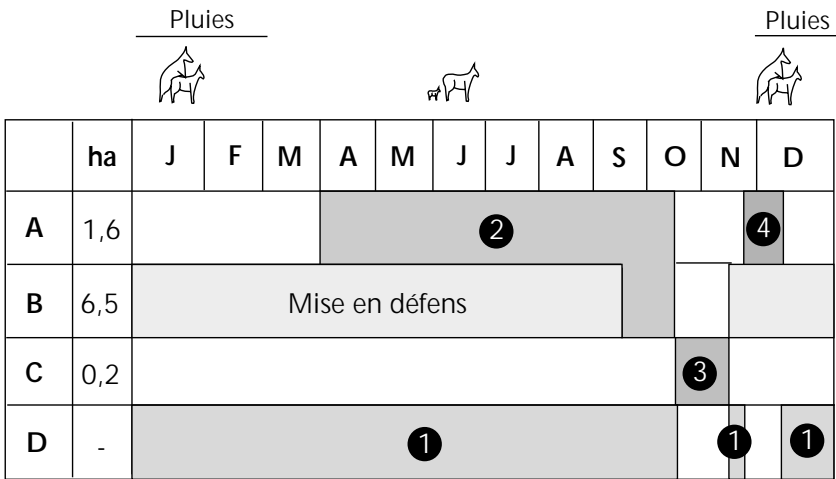

1 Entretien des animaux à coût réduit et appropriation de l'usage des ressources collectives

3 Passer la période de soudure grâce à la suplémentation des animaux

2 Entretien des animaux à besoins élevés et amélioration du cycle reproductif

4) Assurer la saillie dans de bonnes conditions

Figure 4 : chaîne de pâturage de Valdemar en 1991 et objectifs liés aux mouvements des lots. tour à tour la production agricole, la production fourragère et la soudure du calendrier d'alimentation des animaux.

Certaines pratiques, comme le flushing, le contrôle de la lutte, la distribution de foin de pois d'Angole (Cajanus cajan), étaient innovantes. Malgré tout, les performances du troupeau caprin ont été médiocres, comparées à celles des autres éleveurs de Massaroca. Le taux de productivité numérique au sevrage a été de 17,6 p. 100, le taux le plus faible parmi les six éleveurs, suivi par Paris (22) ; certains ont été supérieurs à 100 p. 100 (23).

\section{Pratiques de trésorerie}

Afin de mieux comprendre les raisons qui ont conduit à la vente d'animaux, différents événements ont été mis en perspective : les autres sources de revenus, les dépenses engagées au sein de l'exploitation et de la famille, les pics de travail (figure 5). On constate que Valdemar a dû faire face à de fortes contraintes de trésorerie, principalement en fin de saison sèche (septembrenovembre). La faible pluviométrie enregistrée au cours des années 1990 et les maigres revenus agricoles ont accru les difficultés.

Les raisons ont été multiples. Ses enfants n'étaient pas en âge de travailler et il a dû recourir à l'emploi de main d'œuvre pour clôturer et défricher en saison sèche. Son stock fourrager extrêmement limité l'a souvent contraint à acheter des aliments agro-industriels en période de soudure. Les besoins monétaires, auxquels se sont ajoutés les besoins de la consommation familiale, ont ainsi été considérables en cette période de l'année.

Cette situation a entraîné plusieurs conséquences. Tout d'abord, il a été obligé de travailler hors de l'exploitation, voire de migrer 
Tableau III

Parcellaire et quartiers de caatinga utilisés par Valdemar

(d'après Paris, 1992, M émoire de fin d'études, Eitarc/Cnearc, M ontpellier, France)

\begin{tabular}{|c|c|c|c|c|}
\hline \multicolumn{2}{|c|}{ Parcelle - quartier de caatinga } & \multirow{2}{*}{$\begin{array}{l}\text { Sol } \\
\text { Bruns non calciques }\end{array}$} & \multirow{2}{*}{$\begin{array}{l}\text { Assolement (1990-1991) } \\
\text { Cenchrus ciliaris } \\
\text { et Prosopis juliflora }\end{array}$} & \multirow[t]{2}{*}{ Contraintes pour l'élevage } \\
\hline$A$ & $\begin{array}{l}\text { Parcelle de la Rodagem } \\
\text { (1,6 ha) }\end{array}$ & & & \\
\hline B & $\begin{array}{l}\text { Parcelle des areias } \\
(4,3 \mathrm{ha})\end{array}$ & Régosols & $\begin{array}{l}\text { Pastèques et ricin ( } 3 \text { ha) } \\
\text { Pois d'Angole, maïs et haricots } \\
(0,5 \text { ha) } \\
\text { caatinga ( } 4,2 \text { ha) }\end{array}$ & $\begin{array}{l}\text { Distance du siège d'exploitation } \\
\text { et des parcours }(4 \mathrm{~km}) \\
\text { Transport de l'eau pour } \\
\text { l'abreuvement }\end{array}$ \\
\hline $\mathrm{C}$ & $\begin{array}{l}\text { Parcelle de la maison } \\
(0,2 \mathrm{ha})\end{array}$ & Bruns non calciques & Cenchrus ciliaris & \\
\hline $\mathrm{D}$ & Q uartiers de caatinga & Bruns non calciques & & \\
\hline
\end{tabular}

quelques mois à São Paulo, lorsque la demande locale d'emploi a été pratiquement nulle, comme cela a été le cas en 1993 en raison de la sécheresse. Ces absences ont causé un retard dans la préparation de la campagne agricole suivante et pouvaient entraîner une faible production. Par ailleurs, il a vendu fréquemment des animaux en fin de saison sèche, lorsque leur prix était au plus bas. Il prenait des risques. Compte tenu de la petite taille du troupeau et de la difficulté de prévoir le nombre d'animaux à vendre, il s'y prenait au dernier moment et était alors obligé de vendre des femelles gravides pour subvenir à ses besoins. Le troupeau ne grandissait pas. Les problèmes de trésorerie se répétaient chaque année. Valdemar n'a pas réussi à rembourser son premier emprunt. Grâce au « délai de carence » supplémentaire qui lui a été accordé, il en a même contracté un second.

\section{Interprétation des pratiques}

\section{Motivation des pratiques et critères de décision}

Dans le tableau I, sont présentés quelques exemples de critères de décision relatifs à l'organisation de la chaîne de pâturage, identifiés à partir d'enquêtes portant sur les motivations de mise en œuvre des pratiques observées. Dans le tableau II figurent les cri-

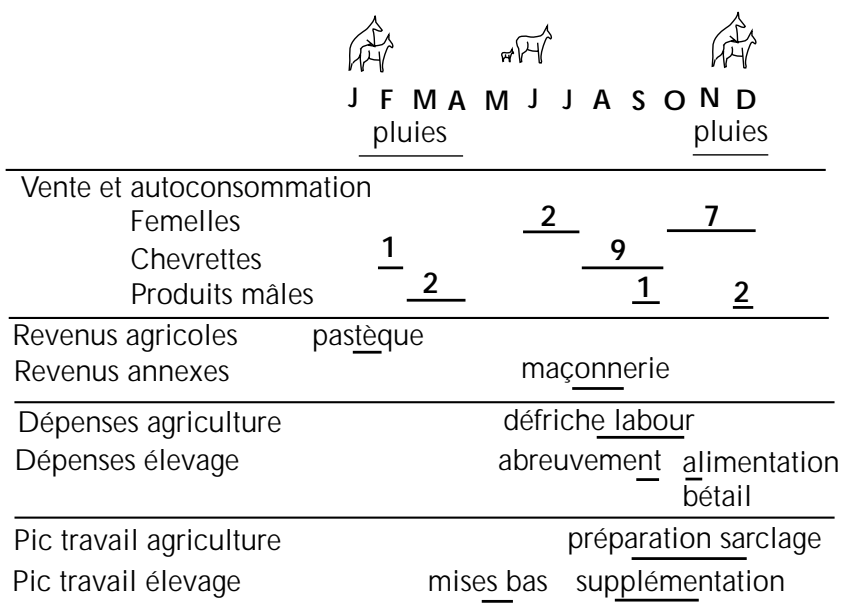

Figure 5 : calendrier des revenus, des dépenses et pics de travail de Valdemar en 1991. tères de décision des pratiques d'élevage de Valdemar. Ce tableau synthétique ne présente que les interprétations des observations et des enquêtes, comme celles qui sont illustrées au tableau I. On y voit par exemple combien les pratiques d'agrégation sont complexes, se caractérisant par un «pilotage à l'individu », possible en raison du petit nombre d'animaux.

Pour chacune des catégories de pratiques relatives à la gestion du troupeau et à celle de l'espace (voir plus haut), le contenu ainsi que les critères qu'utilisait l'éleveur pour appliquer ses règles de décision sont caractérisés. Les auteurs n'ont pas cherché à reconstituer son processus décisionnel, mais à le rendre intelligible à leurs yeux, en identifiant les catégories et les indicateurs que l'éleveur s'était constitués pour piloter son système d'élevage.

\section{Représentation de la gestion du système d'élevage}

La gestion de l'exploitation et du système d'élevage de Valdemar peut être représentée en différenciant quatre phases finalisées qui se sont succédées au cours de la campagne annuelle (figure 6) :

- une première phase où Valdemar a cherché à assurer les revenus de l'année en donnant priorité aux cultures et en valorisant au maximum l'offre fourragère des parcours d'accès libre et gratuit (saison des pluies)

- une deuxième phase où il a investi pour augmenter le capital productif et préparer la saison agricole suivante, tout en assurant une reproduction satisfaisante du troupeau et de bonnes conditions d'alimentation des animaux jusqu'aux pluies suivantes (début de la saison sèche) ;

- une troisième phase où il a cherché à couvrir les besoins de soudure, pour le troupeau et la famille (fin de la saison sèche) ;

- une quatrième phase où il a fallu assurer les besoins de consommation de base de la famille et la survie d'une partie du troupeau.

A chacune de ces phases, correspondaient des objectifs, des stratégies et des pratiques différentes. Ces dernières ont été mises en œuvre en fonction d'objectifs immédiats (production agricole, maintien ou survie du troupeau, consommation de la famille) ou plus lointains (préparation de la saison suivante, accumulation, etc.). L'action - les pratiques - a résulté de choix réalisés par Valdemar à un moment donné en fonction de l'information et de la connaissance technique dont il disposait et des orientations stratégiques retenues pour atteindre ces objectifs spécifiques. 
Phases

1

2

O bjectifs Assurer les
revenus

\section{Stratégies}

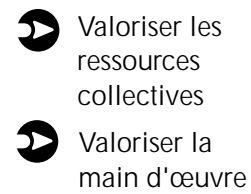

Pratiques

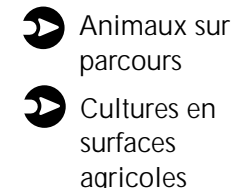

Les stratégies de gestion du territoire de l'exploitation ont été élaborées en fonction de pas de temps pluriannuels. Leur compréhension et leur représentation a donc fait appel à l'analyse du fait technique appréhendé selon plusieurs échelles temporelles.

La succession des quatre phases s'est orchestrée de manière différente chaque année. La durée et la période de chacune d'elles étaient extrêmement variables et dépendaient, entre autres, des conditions climatiques. Il en était de même pour leur intensité. La phase 2, par exemple, pouvait être réduite à la constitution de stocks fourragers de survie, ou, au contraire, se traduire par la clôture d'une superficie importante si les excédents agricoles permettaient l'investissement ou si un financement extérieur était obtenu. La phase 4 n'apparaissait qu'en cas d'année particulièrement défavorable ou lors d'une succession d'années sans excédents. Elle pouvait alors remettre en cause la survie de l'exploitation.

Le champ des possibles diminuait au fur et à mesure que l'année s'écoulait. A chaque phase, les options dépendaient en partie des choix réalisés lors des phases antérieures. $\gg$ main

Assurer le maintien du troupeau en période difficile

Assurerles besoins de consommation de la famille

Mobiliser les stocks fourragers

D

Utilisation des stocks fourragers (foin, palme...)

(Daçonnerie survie du troupeau en période critique

D Assurer les besoins de la famille

$\$$ Mobiliser les réserves monétaires pour acheter des fourrages

D Achat d'aliments du bétail...

( ....grâce à la vente d'animaux

D Maçonnerie et éventuellement migration
Le projet de production et de développement de l'exploitation reposait cependant sur l'enchaînement de plusieurs campagnes se succédant les unes aux autres, non sans liens étroits. On peut ainsi identifier des cycles pluriannuels, constitués de successions d'années de différents types :

- une année sans excédents, au cours de laquelle peu de changements sont apparus :

- une succession d'années sans excédents ou une année particulièrement difficile, entraînant une décapitalisation (diminution du noyau d'animaux reproducteurs) ;

- une année avec excédents, suivant plusieurs années difficiles ; les excédents étaient alors mobilisés pour subvenir aux dépenses retardées au maximum et l'investissement était difficile ;

- une succession de plusieurs années avec excédents, permettant l'investissement et se traduisant par une croissance de la structure d'exploitation (principalement la surface clôturée dans le cas de Valdemar). 
Les périodes d'accumulation, de décapitalisation ou de compensation se succédaient selon l'agencement de ces types d'années. La trajectoire de l'exploitation en était l'expression. L'évolution des structures permet d'en rendre compte (figures 7 et 8). On observe : (i) une diminution de la taille du troupeau après les sécheresses de 1983-1984 (diminution forte) et 1990 ; (ii) une augmentation nette des surfaces clôturées en 1989 et 1994, après l'obtention d'un crédit ; (iii) une évolution peu sensible des structures les autres années.

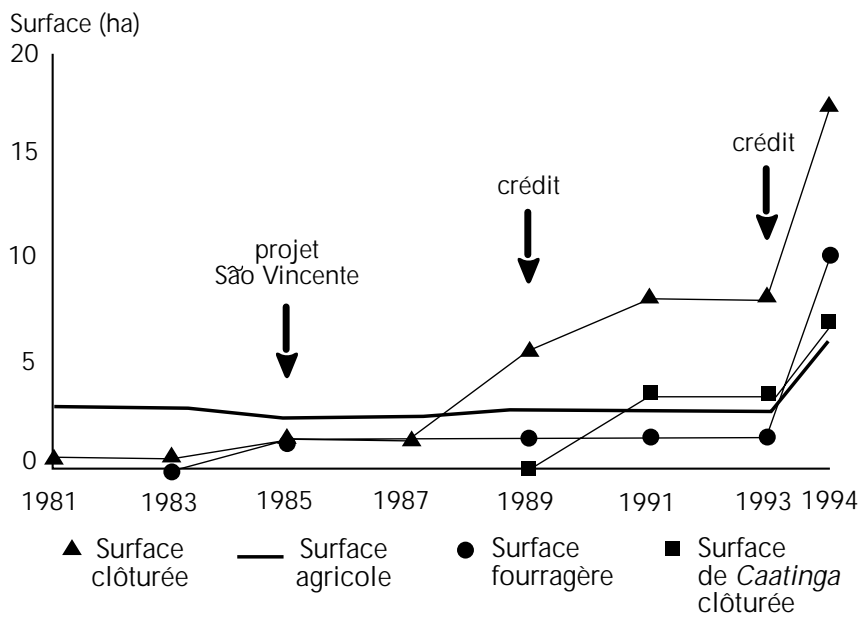

Figure 7 : évolution de la structure foncière de Valdemar.

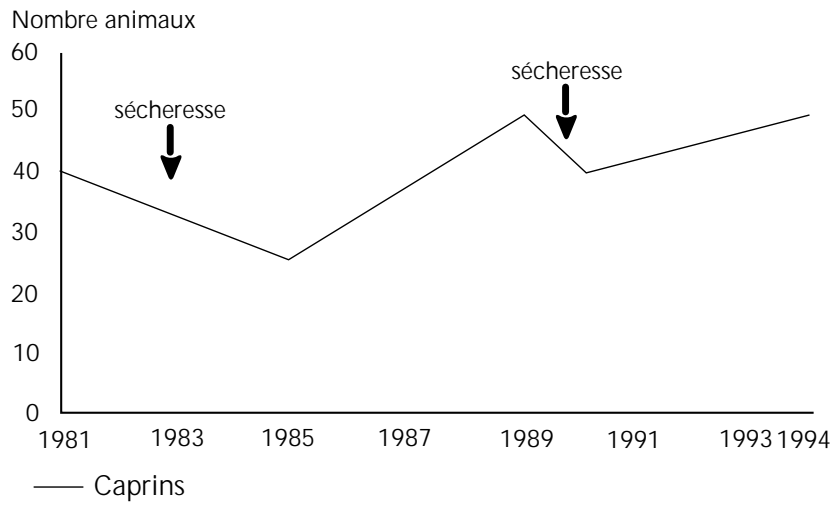

Figure 8 : évolution de la taille du troupeau caprin de Valdemar.

\section{L'analyse stratégique}

Les éléments stratégiques qui ont guidé l'action de Valdemar peuvent être représentés par huit axes définis après l'analyse comparative des stratégies des producteurs de Massaroca (3) (figure 9). Ces huit axes peuvent être regroupés en quatre catégories :

- les modifications portées à la structure foncière (extension foncière et appropriation de ressources d'usage collectif) ; la clôture, et donc l'aménagement foncier, jouent un rôle prépondérant. La délocalisation des productions permise par l'histoire foncière familiale est tout autant une composante de cette stratégie foncière qu'un moyen de valoriser la diversité et la complémentarité des ressources naturelles. Les financements extérieurs et les appuis institutionnels sont recherchés activement ;
- les modifications liées à la mise en valeur des ressources territoriales (mise en place de surfaces fourragères et extension agricole et valorisation de la main d'œuvre) ; les cultures représentent la principale source de revenus pour réaliser les investissements d'aménagement. Il s'agit là d'un pari risqué, mais pouvant conduire à une accumulation plus rapide. La main d'œuvre est valorisée par la mise en œuvre de pratiques exigeantes en travail. La délocalisation et l'extension agricoles s'appuient sur la mise en place de surfaces fourragères ;

- les modifications portées à la structure du troupeau (croît du troupeau et augmentation des ovins et des bovins) ; elle évolue peu, tant en ce qui concerne la taille que les espèces et le type d'animaux qui le composent ;

- la propension à prendre des risques (autosuffisance fourragère et risque économique), c'est-à-dire une prise de risque liée non seulement à l'option agricole retenue, mais aussi à l'endettement chronique et aux migrations fréquentes.

Ces choix se traduisent par des résultats zootechniques médiocres et un croît aléatoire du troupeau malgré la mise en œuvre de pratiques innovantes, par la succession d'années de capitalisation foncière et de décapitalisation liée à la diminution du troupeau. Ce dernier n'en joue pas moins un rôle fondamental, assurant pour l'exploitation des fonctions de consommation, de trésorerie et d'épargne, mobilisée lors des sécheresses. Valdemar et sa famille vivent dans une insécurité persistante, malgré ou étant donné les investissements, la qualification de la main d'œuvre, l'importance des cultures de rente et la technicité démontrée. L'affectation des moyens de production et des produits fait l'objet d'arbitrages constants pour satisfaire à la fois aux besoins de consommation, d'investissement et d'entretien du troupeau.

En conséquence, certaines recommandations standard apparaissent totalement inadaptées au cas de Valdemar, comme, par exemple, la production de légumineuses exclusivement fourragères. Il ne disposait pas d'une surface aménagée pour cela. On comprend au contraire l'importance d'un appui financier adapté à la situation des jeunes en phase d'installation et l'intérêt de thèmes techniques spécifiques (associations de cultures, itinéraires économes en intrants, valorisation des restes de culture pour l'affouragement, etc.).

Valdemar apparaît donc comme un jeune producteur particulièrement dynamique et entreprenant. Il était reconnu comme étant un grand travailleur (22). Il possédait une très forte capacité d'ouver-

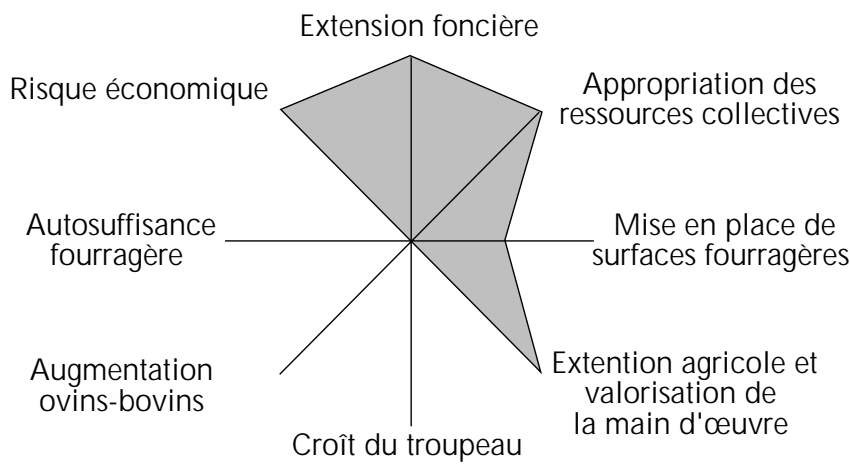

Figure 9 : principaux éléments des stratégies mises en œuvre par Valdemar. Sur chaque axe, la valeur attribuée au critère augmente à mesure que l'on s'éloigne du centre de la figure (Caron P., 1998, Thèse Doct. G éographie, Université Paris X N anterre, France). 
ture sur l'extérieur et savait saisir les opportunités d'aides proposées par les services techniques quand elles se présentaient. Il a clairement défini son projet : clôturer et mettre en valeur les terres d'areias. Il a choisi d'investir dans l'aménagement du foncier, plutôt que de compter sur l'accumulation lente que lui aurait procurée le crôit d'un troupeau conduit extensivement. A terme, il souhaitait même creuser un puits et mettre en place une parcelle d'irrigation. Pour mener à bien ces projets, il a pris des risques, alors que son patrimoine ne lui permettait pas de faire face aux aléas climatiques et que sa force de travail était particulièrement réduite. Il lui a fallu jongler avec des objectifs qui n'étaient pas toujours compatibles : assurer la productivité et le croît du troupeau, garantir l'alimentation de sa famille par la production de cultures vivrières et d'animaux et par l'achat de denrées grâce aux différents revenus agricoles et non agricoles, dégager des excédents pour « coloniser » de nouvelles terres.

Les itinéraires individuels d'éleveurs comme Valdemar ont ensuite été reliés aux transformations de leur environnement ainsi qu'aux évolutions des autres systèmes de production du système agraire local : l'accès à la terre, aux circuits de commercialisation, au crédit, à l'offre technologique et à l'appui technique éventuel ont été abordés au niveau du collectif local. Ces différents éléments ont été étudiés et formalisés afin d'élaborer un modèle général d'évolution des systèmes d'élevage.

\section{DISCUSSIO N}

Le modèle d'évolution des systèmes d'élevage comprend tout d'abord l'identification de types de systèmes. A chacun correspondent des formes d'organisation particulières au niveau de l'exploitation agricole qui ont été caractérisées, pour chaque situation, par un « état » du système. Ces états se traduisent par des modes de gestion et un ensemble de stratégies et de pratiques semblables qui n'évoluent qu'à certaines conditions. Mais le modèle comprend également la caractérisation des processus de transition d'un état à un autre et, en particulier, des seuils et des caps liés à ces transitions, c'est-à-dire, d'une part, les limites au-delà desquelles se mettent en place de nouvelles formes d'organisation, d'autre part, les obstacles à franchir et les conditions à remplir pour passer d'un état à un autre. Ce modèle peut alors se décliner dans une diversité de situations locales, chacune caractérisée par des paramètres démographiques, fonciers, économiques, etc., dépendant de l'histoire de la localité, de son insertion dans un ensemble régional et de son implication dans les dynamiques et les politiques de développement.

\section{Typologie des systèmes d'élevage dans le Nordeste du Brésil}

L'analyse comparative des stratégies des éleveurs de quatre petites régions du Nordeste (figure 1) conduit à la distinction de cinq types d'élevage (encadré 1) (3). La dénomination des trois premiers fait référence aux caractéristiques et au mode d'accès à la ressource fourragère, celle des deux autres à l'espèce animale, au type de production et aux techniques de supplémentation. Le système d'élevage de Valdemar était du type 2.

Dans la situation actuelle de l'élevage dans le Nordeste, ces cinq types peuvent être considérés comme autant d'étapes d'un continuum concernant l'appropriation et la mise en valeur des ressources foncières, passant par une saturation progressive de l'espace libre à l'échelle de la petite région et l'intensification (figure 10). Des chaînes d'évolution technique peuvent être identi- fiées : on ne passe pas directement du type 1 au type 5. La production laitière devient une option pour l'éleveur lorsqu'il dispose déjà de prairies clôturées.

Toutefois, il ne s'agit pas là d'un modèle évolutionniste du développement local et si, de toute façon, les transitions sont progressives, elles ne sont pas inéluctables. De nombreux exemples locaux en témoignent. Il n'empêche que ce mouvement général permet de caractériser une situation locale - également par ses écarts au modèle - et, à partir de cette caractérisation, de raisonner et d'agir localement sur les options possibles de développement, les précautions à prendre et les moyens qu'il faut mobiliser pour agir sur l'avenir. Un tel modèle vise en fait à aider les acteurs et les organisations impliqués à transformer la situation en développant leurs capacités d'autonomie, de conception et de mise en œuvre des voies techniques, institutionnelles et politiques de maîtrise de leur propre devenir.

On ne rencontre jamais tous les types d'élevage dans une même petite région. En effet, le type d'élevage est conditionné en partie par l'environnement dans lequel le producteur évolue. L'organisation locale de la production, la structure foncière, l'organisation de la mise en marché et des filières sont autant de facteurs parmi d'autres qui conditionnent les choix techniques individuels. Ainsi, Caron et Hubert (4), à partir d'une analyse comparative de trajectoires de développement local, ont identifié quatre types d'espace local, à l'échelle d'une petite région économique ayant un sens du point de vue administratif (Municipe) ou sociétal (communauté) : (i) l'espace pionnier où dominent les stratégies individuelles ou collectives d'appropriation foncière ; (ii) l'espace de production diversifiée où une majorité d'exploitations conduisent des activités et élaborent des produits multiples et variés ; (iii) le bassin de production où un nombre significatif d'exploitations sont spécialisées autour du même produit ; (iv) l'espace marginalisé où les productions locales ne permettent pas aux populations de subvenir à leurs besoins, ni de reproduire les facteurs humains et matériels des processus productifs. Dans la partie suivante, la discussion porte sur les interactions entre, d'une part, ces dynamiques collectives induites par les stratifications politiques et économiques régionales et, d'autre part, les transformations des systèmes d'élevage et les conditions d'émergence des différents types qui viennent d'être présentés.

\section{Au cour des transitions entre les types d'élevage, les dynamiques locales et les trajectoires d'évolution de l'exploitation}

Les relations entre les choix techniques qui marquent les transitions entre les types d'élevage et, d'une part, les évolutions de l'espace local, d'autre part, la situation de l'exploitation sur une trajectoire d'évolution sont analysées.

Pour les auteurs, les systèmes d'élevage sont à la fois révélateurs et organisateurs de l'état d'une petite région : les activités d'élevage sont conditionnées par l'accès aux moyens de production, aux équipements et aux services, par les possibilités d'écoulement de la production. Les changements s'appuient sur des processus collectifs de coordination et d'apprentissage. Les activités d'élevage contribuent aussi à la production de nouveaux espaces ou à la stabilité des états qui les caractérisent. Lorsqu'elles évoluent, l'espace se transforme. Il acquiert de nouvelles caractéristiques et il est le siège de nouveaux usages. Les fonctions qui lui sont attribuées changent de nature avec les transformations qualitatives des ressources opérées pour satisfaire aux besoins de l'élevage. Les activités d'élevage peuvent donner naissance à de nouvelles formes d'organisation locale. Le risque de raréfaction des res- 


\section{Encadré 1}

Typologie des systèmes d'élevage dans le Nordeste du Brésil

Type 1 : élevage multi-spécifique (bovins, ovins, caprins) fort utilisateur de parcours collectifs non clôturés La logique d'exploitation maximale d'une ressource végétale collective en accès libre et gratuit domine. Les animaux sont conduits extensivement, lâchés sur parcours de caatinga. Les trois espèces de ruminants sont présentes. Les éleveurs valorisent la diversité et la variabilité de la caatinga dans le temps et dans l'espace. Sauf lors des sécheresses, la charge animale n'est pas une cause de tension. Le temps long de l'accumulation par la croissance numérique du troupeau domine.

Type 2 : élevage multi-spécifique sur parcours clôturés et parcours collectifs non clôturés

L'éleveur combine une logique patrimoniale et anti-aléatoire basée sur le croît du troupeau et la valorisation d'une ressource fourragère en accès libre et une logique d'intensification reposant sur l'amélioration des performances zootechniques. II intègre ces deux logiques par des pratiques d'allotement et de conduite alimentaire spécifiques (à certains stades physiologiques animaux sur des parcours collectifs, à d'autres sur des surfaces fourragères auparavant mises en défens). La clôture à neuf fils s'impose pour empêcher les autres troupeaux de pénétrer dans les parcelles. L'appropriation individuelle des parcours s'accompagne de conflits et d'exclusions sociales. Des formes de gestion collective des parcours reposant sur une réglementation de l'accès à la ressource se dessinent parfois.

Type 3 : élevage multi-spécifique sur prairies artificielles et parcours

Tout l'espace est clôturé, la surface pastorale bornée et la taille du troupeau ne peut augmenter de manière incon- sidérée. La notion de charge animale devient essentielle. Les bovins prennent de l'importance. Pour les contenir, quatre fils suffisent. L'éleveur maîtrise le choix des reproducteurs et le déroulement du cycle reproductif. La gestion des ressources hydriques structure l'organisation territoriale de l'exploitation.

Type 4 : élevage bovin à viande sur prairies artificielles

La caatinga est remplacée par des prairies de graminées, les bovins dominent et les caprins ont disparu. La gestion de la pérennité des prairies est capitale. L'état de la ressource végétale devient un critère de décision majeur pour l'organisation du calendrier et de la chaîne de pâturage. Ce système, peu exigeant en main d'œuvre, est aisément géré par des propriétaires absentéistes. La croissance de l'exploitation s'opère par l'achat de terres et l'augmentation consécutive de l'effectif du troupeau.

Type 5 : élevage bovin mixte (laitier et à viande)

La production laitière se développe, mais la production de viande demeure importante. La gestion de l'exploitation est marquée par des itinéraires techniques exigeants en main d'œuvre et un investissement important (génétique et infrastructures). L'organisation territoriale de l'exploitation devient essentielle pour faciliter la mise en œuvre des pratiques d'allotement et de conduite. Pour augmenter la production laitière, garantir sa régularité au cours de l'année, diminuer les coûts de production liés à l'achat d'aliments pour le bétail, une seconde intensification fourragère fait suite à l'installation des prairies. Elle correspond à des situations techniques et géographiques différentes et s'appuie, soit sur la production de fourrage en irrigué, soit sur la production d'ensilage de maïs. sources pastorales est à l'origine, à Massaroca, d'associations qui, ensuite, ont assuré des fonctions dépassant le cadre de l'élevage. Les sociétés locales, en s'appropriant des ressources pastorales, en changeant d'espèce animale, de race ou de production, créent des institutions, des règles d'action. En un mot, elles transforment leur espace d'action en un territoire collectif organisé.

Cependant, l'influence de l'espace local n'explique pas tous les changements. Le passage d'un type d'élevage à un autre nécessite la mobilisation de moyens de production, l'apprentissage et la maîtrise de nouvelles techniques et de nouvelles formes d'organisation. La position de l'exploitation sur une trajectoire d'évolution joue, comme nous le verrons, un rôle majeur.

\section{Choix techniques et dynamiques locales}

Les trois premiers types d'élevage sont marqués par l'expression de logiques pionnières. Le premier type correspond aux systèmes d'élevage qui ont accompagné la colonisation du Nordeste. Il subsiste dans les zones enclavées, au sein de grandes exploitations d'élevage extensif, ou, comme à Massaroca, là où les parcours sont importants. La pression démographique ou foncière peut conduire à l'élaboration de stratégies d'appropriation individuelle des ressources collectives. Certaines exploitations évoluent alors vers le deuxième type. Franchir ce cap correspond à un choix stra- tégique de l'exploitant qui opte alors pour un changement de type plutôt que pour la croissance du nombre d'animaux.

Lorsque le second type se généralise, l'appropriation individuelle des parcours collectifs est stratégique. Sauf lorsque sont définies et respectées des règles d'accès aux ressources collectives (24), la situation incite les éleveurs à clôturer plus et plus vite, accélérant l'évolution vers le type 3 , où l'ensemble des terres est clôturé.

Le troisième type marque l'aboutissement des logiques d'appropriation individuelle. L'augmentation des rendements par unité de surface ou de bétail reste limitée. Les logiques patrimoniales mobilisent l'essentiel des moyens de production. La croissance de l'exploitation s'opère par l'achat de nouvelles terres ou par l'intensification fourragère grâce à la mise en place de prairies artificielles et de points d'eau. Le type 3 comprend en particulier des exploitations de plusieurs milliers d'hectares, les fazendas, pratiquant un élevage bovin extensif, avec peu d'intrants et de main d'œuvre *. Pour les producteurs familiaux, les revenus de l'élevage ne suffisent pas et la diversification des activités est nécessaire. L'installation de prairies marque la transition vers le type 4 . Les types 3 et 4 sont fréquents dans les espaces de production diversifiée.

* Il s'agit de types fonctionnels, et non structurels. Il n'est donc pas étonnant d'y rencontrer des exploitations de tailles différentes 


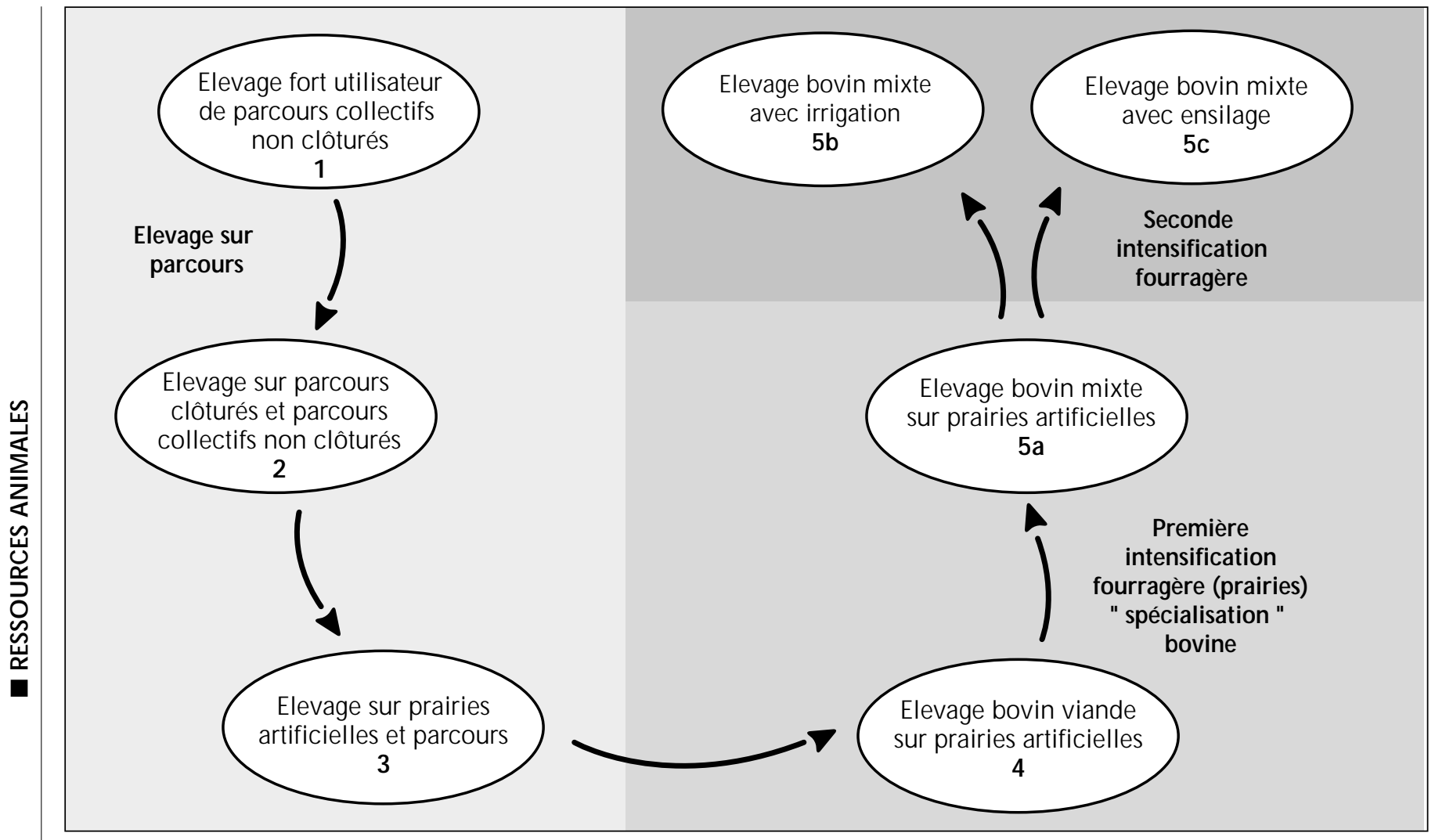

Figure 10 : types de systèmes d'élevage dans le N ordeste et transitions.

Quand s'opère la transition vers l'état de bassin de production laitière, le type 5 se généralise, même si de nombreux fazendeiros maintiennent des systèmes d'élevage de type 3 ou 4. L'accès aux intrants, aux équipements et aux services, l'organisation des circuits commerciaux, la constitution de réseaux d'apprentissage sont autant de facteurs qui favorisent la reconversion des exploitations. Lorsque se structure un marché local de commercialisation de lait ou de fromage, le risque de ne pas pouvoir écouler la production diminue. De nombreuses exploitations se reconvertissent. Lorsque l'investissement est facilité, comme à Gloria par l'octroi d'aides financières ou à Tauá et Pintadas par la construction de retenues collinaires, cette reconversion est rapide.

L'importance des logiques patrimoniales en situation d'espace pionnier ou la reconversion des exploitations lors de la formation d'un bassin de production laitière illustrent la marque de l'espace sur les exploitations et les activités d'élevage. Les changements observés sont marqués par des seuils. Trois semblent majeurs. Le premier est lié à l'appropriation des ressources foncières. La généralisation de la propriété individuelle, matérialisée par la clôture, a marqué la colonisation. On observe encore une résurgence de phénomènes comparables dans les espaces où il existe des communs. Ces processus se prêtent aux conflits et à l'émergence de formes particulières d'organisation politique et sociale visant à faciliter l'appropriation, à combattre les envahisseurs ou, parfois, à réguler l'accès à des ressources appelées à demeurer collectives, pour un temps au moins. Le second seuil est lié à l'utilisation productive des ressources hydriques. Pour cela, il faut résoudre les problèmes d'approvisionnement domestique, s'organiser pour revendiquer les transferts financiers et les infrastructures. La gestion du risque change de nature. Des poches d'intensification de la production apparaissent. Le champ de la diversification s'ouvre conjointement à l'organisation de circuits commerciaux. Le troisième seuil cor- respond à l'entrée des opérateurs de la filière laitière dans l'arène locale (collecteurs, transformateurs artisanaux ou industriels, commerçants, etc.). L'espace local est intégré à un bassin de production aux dimensions organisationnelles nouvelles, du fait de la relation avec des acteurs faisant jouer la concurrence nationale, voire internationale.

Ces seuils sont bien ceux « à partir desquels apparaissent, dans un milieu donné, des phénomènes nouveaux : des services et équipements rares, des transports collectifs, etc. ; des possibilités d'agir ; sans doute des comportements » (2). En les franchissant, la nature et la cohésion des systèmes évoluent (4).

\section{Choix technique et trajectoire d'exploitation}

La rapidité et l'ampleur de l'accumulation dépendent du capital disponible à l'installation, de l'évolution de la composition de la famille et de la main d'œuvre mobilisable. On retrouve l'un des traits du fonctionnement des exploitations agricoles familiales, l'existence d'un cycle de vie de la famille au cours duquel évoluent l'investissement en travail et les choix d'affectation des revenus entre consommation, investissement et épargne (26).

Les trajectoires sont marquées par la succession de trois phases. La première, l'installation, conditionne les évolutions ultérieures. Période comprise entre le moment où le producteur commence à gérer certains moyens de production et celui où il gère de manière autonome les moyens nécessaires à la reproduction de sa famille, cette installation est souvent longue et progressive. On peut distinguer trois cas de figure : (i) l'installation est immédiate, lors d'un héritage ou d'une donation ; (ii) elle fait suite à une migration urbaine et à l'épargne, ce qui est le cas de la majorité des petits producteurs à l'époque du «miracle économique »; (iii) elle s'effectue après que le producteur a été métayer, souvent sur 
l'exploitation de son père où il a élevé son propre troupeau qu'il a vendu en partie pour dégager les fonds nécessaires.

La seconde phase, la « croisière », est marquée par la recherche d'accumulation, parfois hors du secteur agricole. Lorsque des terres en accès libre existent, l'accumulation s'appuie sur le crô̂t du troupeau élevé sur parcours ou sur la clôture de terres. Le troupeau est un capital productif et un patrimoine mobilisable pour investir. Dans le cas contraire, c'est-à-dire quand il n'y a plus de terres en accès libre, deux voies plus ou moins complémentaires peuvent être suivies selon les choix d'affectation des ressources :

- le croît de la productivité de l'élevage. Le capital d'exploitation augmente (valeur des animaux, équipements, infrastructures). La charge animale par unité de surface croît de manière continue, passant de 0,3 unité animale (UA *) par hectare lorsque l'alimentation est fournie par les prairies à plus d'1 UA/ha lorsque les animaux sont supplémentés. Les changements techniques sont importants : l'évolution de la gestion de l'exploitation repose sur des processus d'apprentissage, la mobilisation de nouveaux matériels biologiques (espèce, race) et de moyens organisationnels et financiers, la constitution de réseaux professionnels ;

- l'extension sans changement technique majeur. L'extension foncière et le croît du nombre d'animaux allant de pair ne modifient pas directement le chargement animal par unité de surface. Ainsi la charge animale ne correspond pas à un critère de gestion technique, mais elle reflète la position de l'exploitation sur la trajectoire d'accumulation. Avant l'achat d'une nouvelle parcelle, elle peut augmenter de manière importante, parfois dangereusement pour l'état de la ressource pastorale. A l'inverse, après l'achat de terre, la vente d'animaux et l'augmentation de la surface entraînent une baisse significative de la charge.

La troisième phase, la transmission, se réalise par héritage ou par cession aux enfants du vivant des parents. Dans ce dernier cas, les parents accordent à un enfant une parcelle et/ou des animaux. La donation peut être retardée au maximum par le père, qui dispose alors d'une main d'œuvre abondante. Il « retient » d'autant plus facilement ses enfants que l'accès au foncier est difficile.

Au fil de l'évolution d'une exploitation, les fonctions économiques et sociales des productions évoluent. Comme dans le cas de Valdemar, les choix techniques sont étroitement liés à la position de l'exploitation sur une trajectoire. Dans son cas, la priorité a été donnée aux productions végétales de vente. La très faible productivité du troupeau n'est pas considérée comme un problème grave tant qu'elle ne remet pas en cause de manière trop importante la survie d'un noyau de mères et le potentiel de croissance de l'effectif à un terme relativement éloigné. La sécurisation repose alors sur l'amélioration de quelques parcelles pastorales, insuffisantes toutefois pour agir significativement sur le relèvement du niveau des performances zootechniques.

L'identification de ces trajectoires est importante pour la mise en œuvre d'actions de développement. Thèmes techniques et systèmes d'aides sont différents selon le producteur et selon sa position sur la trajectoire d'exploitation. Un jeune en phase d'installation sera réceptif aux conseils et aux appuis allant dans le sens d'un croît rapide du troupeau (amélioration du taux de fécondité, etc.), alors que des éleveurs spécialisés s'intéresseront avant tout à l'augmentation de la productivité laitière ou bouchère par animal (amélioration génétique, intensification fourragère, etc.).

\footnotetext{
* Le système de calcul brésilien est ici utilisé : un taureau ou un bœuf adulte correspond à $1 \mathrm{UA}$, une vache (en moyenne $250-300 \mathrm{~kg}$ ) à $0,9 \mathrm{UA}$, un taurillon ou une génisse à $0,7 \mathrm{UA}$, un veau à $0,4 \mathrm{UA}$, un petit ruminant adulte à $0,15 \mathrm{UA}$
}

\section{- CONCLU SION}

L'entrée par l'étude des systèmes d'élevage et de leurs transformations, en relation avec les dynamiques de développement, illustre les interactions, dans la situation du Nordeste brésilien, entre le changement technique et les modes d'appropriation et de mise en valeur des ressources foncières. Dans de telles situations de mutations rapides, les cycles de vie des exploitations apparaissent comme déterminants pour l'évolution de l'élevage.

Les transformations des systèmes d'élevage sont ainsi très liées au contexte local dans lequel elles se réalisent et donc aux actions des autres opérateurs du développement agricole et rural : formes de coopération ou de compétition avec les autres exploitations ; relations, formelles ou non, avec les acteurs de l'approvisionnement et de la commercialisation des produits ; politiques et projets mis en place par les opérateurs publics ; etc. Des seuils de transformation peuvent ainsi être identifiés. Ils sont autant liés aux dynamiques d'organisation des espaces ruraux qu'aux stratégies individuelles des éleveurs, ce qui conduit les auteurs à affirmer que les systèmes d'élevage peuvent être considérés comme révélateurs et organisateurs des espaces. Ils sont ainsi au cœur de l'inscription territoriale des processus locaux d'innovation.

Pour les auteurs, l'identification et l'interprétation de l'innovation requièrent l'analyse explicite de processus aussi bien locaux que globaux et l'intégration de différents pas de temps : ceux des processus de production eux-mêmes, ceux de l'élaboration des connaissances, ceux de la construction des représentations et ceux de l'action sur ces mêmes processus (11).

Pour ce faire, les auteurs ont élargi le champ des pratiques considérées. Une attention particulière a été accordée aux pratiques territoriales des systèmes d'élevage pour rendre compte de l'empreinte de l'espace et permettre l'intégration, dans l'analyse, d'échelles spatiales qui ont un sens au regard du changement.

A partir de l'analyse des pratiques, l'identification des modalités et des facteurs d'évolution des activités d'élevage repose sur un travail de modélisation. Le modèle d'évolution des systèmes d'élevage proposé comprend l'identification de types de systèmes, mais aussi la caractérisation des processus de transition d'un type à un autre. C'est ainsi que, par une recherche de formalisation, chaque étude de cas a pu être analysée hors de son caractère anecdotique, sans pour autant nier qu'elle était bien liée à un contexte précis, historiquement construit. C'est la démarche d'abstraction, nécessaire à tout travail de modélisation, qui a permis de donner un caractère génératif aux résultats obtenus dans chaque étude de cas. C'est enfin par la modélisation que la dimension temporelle a pu être intégrée à l'analyse, en mettant en évidence les différents pas de temps qui, eux aussi, ont un sens au regard du changement. Cette formalisation ne se limite pas aux seules connaissances in situ produites pendant la durée des observations, mais repose sur la reconstitution des processus analysés à partir des enquêtes.

Les données modélisées ont été établies à partir d'observations et d'enquêtes sur les pratiques des éleveurs et donc sur ce qui était, pour eux, pertinent pour piloter les systèmes d'élevage. Ces données ne concernent pas a priori les mêmes éléments que ceux qui constituent les corpus habituels des connaissances scientifiques. Il a souvent fallu, au cours de ces recherches, appliquer les méthodes scientifiques à des objets nouveaux articulant des processus biologiques aux modalités de gestion de ces processus par les éleveurs. Ces objets ont bien une base matérielle biologique, mais celle-ci se charge de nouvelles qualités tout au long des procès qui organisent la production, au niveau des éleveurs individuellement, mais éga- 
lement au niveau des diverses formes de coordination des éleveurs entre eux autant qu'avec les autres opérateurs des filières de commercialisation et de transformation de leurs produits (11). Plutôt que sur la description d'états et de performances techniques ou économiques, ces recherches ont caractérisé les modes d'action sur les flux, les processus et les changements d'état. Plutôt que sur la constatation de faits mis en évidence, ces travaux ont recherché les cohérences qui donnent un sens aux systèmes d'élevage et aux projets de ceux qui les pilotent. C'est ainsi que peuvent être identifiées des catégories à la fois pertinentes pour l'action et significatives scientifiquement.

\section{BIBLIOGRAPHIE}

1. BONNAL P., CHAIB FILHO H., MADEIRA J.S.N., PANIAGO JR. E., SANTOS M.A. DE, SOUZA G.L.C. DE, SPERRY S., ZOBY J.L.F., 1994 Síntese do projeto Silvânia (1986-1994). Documento preparatorio à avaliação. Montpellier, France, Cirad-sar, Prefeitura Silvânia, Brésil, EM BRAPA-CPAC, $86 \mathrm{p}$.

2. BRUNET R., 1990. Mondes nouveaux : géographie universelle. Paris, France, Hachette/Reclus, $551 \mathrm{p}$.

3. CARON P., 1998. Espace, élevage et dynamique du changement Analyse, niveaux d'organisation et action. Le cas du Nordeste semi-aride du Brésil. Thèse D oct. Géographie, U niversité Paris $X, N$ anterre, France, $396 \mathrm{p}$.

4. CARON P., HUBERT B., 1998. Changement technique et dynamiques locales : le cas de l'élevage dans le N ordeste du Brésil. In : Proc. 15th International Symposium of AFSR/E, Rural Livelihoods, Empowerment and the Environment, November 29-D ecember 4, 1998, Pretoria, South Africa, 3: (non précisées).

5. CHAUVEAU J.P., 1997. Des « stratégies des agriculteurs africains » au " raisonnement stratégique ». Histoire, usages et remise en question d'un concept pluri-disciplinaire. In : Blanc-Pamard et Boutrais, Coord., Thème et variations, Nouvelles recherches rurales au Sud. Paris, France, O rstom, p. 179-218.

6. DURU M., PAPY F., SO LER L.G., 1988. Le concept de modèle général et l'analyse du fonctionnement de l'exploitation agricole. C. R. Acad. Agric. Fr., 74 : 81-93.

7. ETENE, 1964. Recursos e necessidades do Nordeste. Um documento básico sôbre a Região Nordestina. Escritório Técnico de Estudos Econômicos do Nordeste. Recife, Brésil, Banco do Nordeste do Brasil, $666 \mathrm{p}$.

8. GIRARD N., HAVET A., CHATELIN M.H., GIBON A., HUBERT B., RELLIER J.P., 1994. Formalisation des relations entre stratégie et pilotage dans les systèmes fourragers. In : Actes du symposium Recherchessystème en agriculture et développement rural (AFSR/E), Montpellier France, 21-27 novembre 1994. Montpellier, France, Cirad-sar, p. 223 229.

9. HUBERT B., 1991. Comment raisonner de manière systémique I'utilisation du territoire pastoral ? In : IV congrès des Terres de parcours, n̂. Montpellier, France, avril 1991. Montpellier, France, Association $\hat{m}$ française de pastoralisme.

$\because \quad$ 10. HUBERT B., 1994. Pastoralisme et territoire. Modélisation des pratiques d'utilisation. Cah. Agric., $3:$ : 9-22

11. HUBERT B., 1998. Court terme vs long terme: une démarche systémique pour une agriculture durable. In : Proc. 15th International Symposium of AFSR/E, Rural Livelihoods, Empowerment and the Environment, N ovember 29-D ecember 4, 1998, Pretoria, South Africa, 3 (non précisées).
12. HUBERT B., GIRARD N., LASSEUR J., BELLON S., 1993. Les systèmes d'élevage préalpins. Derrière les pratiques, des conceptions modélisables. In : Landais E., Balent G., Ed., Pratiques d'élevage extensif : identifier, modéliser, évaluer. Etud. Rech. Sys. agraires Dév., $27: 351-385$

13. IBGE, 1991. Censo Agropecuário. Instituto Brasileiro de Geografia e Estátisticas. Rio de Janeiro, Brésil, Instituto Brasileiro de Estatísticas e Geografia.

14. LANDAIS E., 1992. Principes de modélisation des systèmes d'élevage. Approches graphiques. Cah. Rech. Dév., 32 : 82-95.

15. LANDAIS E., 1994. Système d'élevage : d'une intuition holiste à une méthode de recherche, le cheminement d'un concept. In : Blanc-Pamard et Boutrais, Coord., A la croisée des parcours. Pasteurs, éleveurs, cultivateurs. Paris, France, O rstom, p. 15-49.

16. LANDAIS E., DEFFONTAINES J.P., 1990. Les pratiques des agriculteurs : point de vue sur un courant nouveau de la recherche agronomique. In : Actes du séminaire Modélisation systémique et systèmes agraires - Décision et organisation, département de Recherche Inra/Sad, St M aximin. Paris, France, Inra/Sad, p. 31-64.

17. LANDAIS E., LHOSTE P., MILLEVILLE P., 1987. Points de vue sur la zootechnie et les systèmes d'élevage tropicaux. Cah. Sci. hum., 23 : 421-437.

18. LEGAY J.M., 1997. L'expérience et le modèle. Un discours sur la méthode. Paris, France, Inra, 111 p. (coll. Sciences en questions)

19. LE MOIGNE J.L., 1990. La modélisation des systèmes complexes. Paris, France, Dunod, $178 \mathrm{p}$.

20. LHOSTE P., 1984. Le diagnostic de système d'élevage. Cah. Rech. Dév., $n^{\circ} 3-4:$ : 84-88.

21. MINTZBERG H., 1987. The strategy concept: Five Ps for strategy, another look at why organizations need strategies. Calif. Manage. Rev., Fall 1987: 11-32.

22. PARIS Y., 1992. Etude des pratiques et stratégies des éleveurs à Massaroca. Mémoire de fin d'études, Eitarc/Cnearc, Montpellier, France, $164 \mathrm{p}$.

23. QUIRIN R., 1993. Epidemologia e prevenção do aborto em caprinos. Relatório de consultoria, Agosto de 1990 - Agosto de 1993. Petrolina, Brésil, CPATSA/EM BRAPA, $60 \mathrm{p}$

24. SABOURIN E., CARON P., DA SILVA P.C.G., 1997. Enjeux fonciers et gestion des communs dans le Nordeste du Brésil : le cas des vaines pâtures dans la région de Massaroca-Bahia. Cah. Rech. Dév., 42 : 5-27.

25. SEBILLOTE M., SOLER L.G., 1990. Les processus de décision des agriculteurs. In : Actes du séminaire M odélisation systémique et systèmes agraires - Décision et organisation, département de Recherche Inra/Sad, St M aximin. Paris, France, Inra/Sad, p. 93-118.

26. TCHAYANOV A., 1990. L'organisation de l'économie paysanne (traduction en français). Paris, France, Librairie du Regard, 344 p.

27. THERY H., 1995. Le Brésil, 3e éd. Paris, France, Masson, 265 p.

28. TONNEAU J.P., CLOUET Y., CARON P., 1997. L'agriculture familiale au Nordeste (Brésil). Une recherche par analyses spatiales. $N$ at. Sci. Soc., 5 : 39-49.

29. YUNG J.M., ZASLAVSKI J., 1992. Pour une prise en compte des stratégies des producteurs. Montpellier, France, Cirad-sar, 72 p. (coll. Documents systèmes agraires $n^{\circ} 18$ )

Reçu le 28.02.00, accepté le 21.09 .00 


\section{Summary}

Caron P., Hubert B. From analyzing practices to designing an evolution model for animal husbandry systems: application to the $\mathrm{N}$ ordeste region of Brazil

A methodological approach based on the analysis of livestock farmers' practices was used to understand the regional evolution of animal husbandry systems. The concept framew ork is presented in the first part. The methodology used to concretize this approach is described in the second part. It is illustrated in the third part by the case-study of a livestock farmer's practices in the Nordeste region of Brazil. Finally, to develop this approach the authors proposed and discussed an evolution model for animal husbandry systems in the area, designed on the basis of a comparative analysis of the farmers' practices and strategies. Identification and interpretation of change required the explicit analysis of local as well as global processes, and the integration of time-scales. To do so, special attention was paid to territorial management practices of the animal husbandry systems. They helped specify the influence of space and integrate spatial scales in the analysis, which were meaningful with regard to change. Based on an analysis of the practices, identification of evolution factors and modes of livestock farming lay in a modeling process, whose results are discussed.

Key words: Animal husbandry methods - Model - System analysis - Livestock management - Nordeste - Brazil.

\section{Resumen}

Caron P., H ubert B. Del análisis de las prácticas a la construcción de un modelo de evolución de los sistemas de crianza: aplicación a la región Nordeste de Brasil

En el presente articulo se describe una maniobra de metodología cuyo objetivo es la comprensión de la evolución regional de los sistemas de crianza a partir del análisis de las prácticas de los criadores. Después de presentar un cuadro conceptual en la primera parte, se describe en la segunda parte, la metodología utilizada para poner en marcha esta maniobra. En la tercera parte, esta se ilustra mediante un estudio de casos, sobre el análisis de las prácticas de un criador de la región N ordeste de Brasil. Finalmente, los autores desarrollaron esta maniobra proponiendo y discutiendo un modelo de la evolución de los sistemas de crianza en esta región, construido a partir del análisis comparativo de las prácticas y de las estrategias de los criadores. La identificación y la interpretación del cambio exigieron un análisis explícito del proceso, tanto local como global, así como la integración de diferentes pasos de tiempo. Para esto, se dio particular atención a las prácticas territoriales de los sistemas de crianza, para evidenciar la marca del espacio y permitir la integración, en el análisis, de las escalas espaciales que presentaban un sentido con respecto al cambio. A partir del análisis de las prácticas, la identificación de las modalidades y de los factores de evolución de las actividades de crianza, se basó sobre un trabajo de modelos, cuyos resultados se discuten.

Palabras clave: Método de crianza - Modelo - Análisis de sistemas - Manejo del ganado - Nordeste - Brasil. 